\title{
Comparison of the accuracy and proximal shunt failure rate of freehand placement versus intraoperative guidance in parietooccipital ventricular catheter placement
}

\author{
Thomas J. Wilson, MD, ${ }^{1}$ Kathleen E. McCoy, MD, ${ }^{2}$ Wajd N. Al-Holou, MD, ${ }^{1}$ Sergio L. Molina, BS, ${ }^{2}$ \\ Matthew D. Smyth, MD, ${ }^{2}$ and Stephen E. Sullivan, MD ${ }^{1}$ \\ 1Department of Neurosurgery, University of Michigan, Ann Arbor, Michigan; and 2Department of Neurosurgery, Washington \\ University in St. Louis, Missouri
}

\begin{abstract}
OBJECTIVE The aim of this paper is to compare the accuracy of the freehand technique versus the use of intraoperative guidance (either ultrasound guidance or frameless stereotaxy) for placement of parietooccipital ventricular catheters and to determine factors associated with reduced proximal shunt failure.

METHODS This retrospective cohort study included all patients from 2 institutions who underwent a ventricular cerebrospinal fluid (CSF) shunting procedure in which a new parietooccipital ventricular catheter was placed between January 2005 and December 2013. Data abstracted for each patient included age, sex, method of ventricular catheter placement, side of ventricular catheter placement, Evans ratio, and bifrontal ventricular span. Postoperative radiographic studies were reviewed for accuracy of ventricular catheter placement. Medical records were also reviewed for evidence of shunt failure requiring revision. Standard statistical methods were used for analysis.
\end{abstract}

RESULTS A total of 257 patients were included in the study: 134 from the University of Michigan and 123 from Washington University in St. Louis. Accurate ventricular catheter placement was achieved in $81.2 \%$ of cases in which intraoperative guidance was used versus $67.3 \%$ when the freehand technique was used. Increasing age reduced the likelihood of accurate catheter placement (OR $0.983,95 \% \mathrm{Cl} 0.971-0.995 ; p=0.005)$, while the use of intraoperative guidance significantly increased the likelihood $(\mathrm{OR} 2.809,95 \% \mathrm{Cl} 1.406-5.618 ; p=0.016)$. During the study period, 108 patients $(42.0 \%)$ experienced shunt failure, 79 patients $(30.7 \%)$ had failure involving the proximal catheter, and 53 patients $(20.6 \%)$ had distal failure (valve or distal catheter). Increasing age reduced the likelihood of being free from proximal shunt failure (OR $0.983,95 \% \mathrm{Cl} 0.970-0.995 ; p=0.008$ ), while both the use of intraoperative guidance (OR 2.385, 95\% $\mathrm{Cl} 1.227-5.032 ; p=0.011)$, and accurate ventricular catheter placement (OR 3.424, 95\% $\mathrm{Cl} 1.796-6.524 ; p=0.009$ ) increased the likelihood.

CONCLUSIONS The use of intraoperative guidance during parietooccipital ventricular catheter placement as part of a CSF shunt system significantly increases the likelihood of accurate catheter placement and subsequently reduces the rate of proximal shunt failure.

http://thejns.org/doi/abs/10.3171/2016.5.FOCUS16159

KEY WORDS cerebrospinal fluid; ventricular catheter; ventriculoperitoneal shunt

$\mathrm{C}$ EREBROSPINAL fluid diversion by way of ventriculoperitoneal (or other terminus) shunting is a commonly performed neurosurgical procedure but one that is fraught with high rates of failure. Up to one-third of adult patients undergoing CSF shunting will experience a shunt failure. ${ }^{8}$ Mechanical obstruction is the most common reason for failure, and in proximal catheter failure, this typically means obstruction by the choroid plexus. ${ }^{4,11}$
Optimal catheter placement may help reduce the rate of proximal failure. We have previously shown that the use of intraoperative guidance with either frameless stereotaxy or ultrasonography improves the accuracy of catheter placement, which in turn reduces the rate of proximal failure in adult patients. ${ }^{12}$ This is supported by previous data suggesting that endoscopic placement of ventricular catheters in pediatric patients reduced the risk of proximal 
failure, although it did not have any significant effect on overall failure rate. ${ }^{11}$ Taken together, these data suggest the importance of optimal catheter placement in reducing proximal failure. Our previous data were limited to adult patients and predominantly frontal catheters, thereby limiting generalizability to pediatric patients and to parietooccipital catheters. The current study was intended to address this issue of generalizability by specifically including both adult and pediatric patients and focusing on parietooccipital catheter placement. It is not clear whether, when utilizing a parietooccipital approach, it is inherently more difficult to achieve accurate catheter placement or whether the trajectory of the catheter or ultimate target for the catheter tip is any more or less likely to lead to proximal catheter failure. The purpose of this study was to compare the accuracy of freehand placement versus use of intraoperative guidance in parietooccipital ventricular catheter placement and to further compare the proximal failure rates with these methods of placement.

\section{Methods \\ Study Design}

This cohort study was approved by both the University of Michigan and Washington University in St. Louis institutional review boards, and data were obtained by retrospective review of medical records, including radiographic images. Medical record databases were queried by information technology personnel to identify all patients who underwent creation of a CSF shunt between January 2005 and December 2013. All patients underwent a preliminary review to confirm that a CSF shunt operation was performed. A secondary review was then performed to identify only those patients who underwent placement of parietooccipital catheters. Only patients who underwent new parietooccipital shunt placement were included. Any patient who underwent frontal catheter placement was excluded. Similar to our previous study, a "new" shunt was considered to be any shunt for which a ventricular catheter was passed into the ventricular system in a new location and not through an existing tract. ${ }^{12}$ The index of radiographic studies was then reviewed for each patient, and patients were included if they had undergone CT scanning or MRI of the head within 48 hours prior to the operation and within 48 hours following the operation. Any patient who did not meet this criterion was excluded.

For the included patients, a detailed chart review was performed, and the following data were abstracted: age, sex, side of shunt, bifrontal ventricular span (based on preoperative imaging findings), Evans ratio (based on preoperative imaging), accuracy of ventricular catheter placement (based on postoperative imaging), and whether intraoperative guidance was used for placement. Both institutions used the StealthStation AxiEM (Medtronic) with a navigated catheter introducer for placement of catheters with frameless stereotaxy. The bifrontal ventricular span was measured as the width of the lateral margin of the right frontal horn to the lateral margin of the left frontal horn at its widest point on an axial image. The Evans ratio was calculated by dividing the bifrontal ventricular span by the widest width of the inner table of the cranium. As we defined it previously, an "accurate" ventricular catheter placement was credited when the tip of the ventricular catheter resided within the intended ventricle. ${ }^{12}$ Additionally, catheter placement was considered inaccurate if the entry point into the ventricular system was anywhere other than the posterior horn of the lateral ventricle and if the tip of the catheter resided in the temporal horn of the lateral ventricle rather than within the frontal horn. All catheters in this study were intended for the ipsilateral ventricle. The accuracy of each ventricular catheter placement was determined by comparing the operative approach to the documented tip position in each radiology report from the postoperative imaging study. Each postoperative imaging study was then reviewed by a member of the study team to confirm that our assessment of the catheter tip was in agreement with the assessment documented in the radiology report. In all patients, the assessment of the study team was in accord with the radiology report.

The medical record was then reviewed for documented evidence of shunt failure. Shunt failure was defined as the necessity for operative intervention to explore/revise the shunt. Shunt exploration/revision was performed at the discretion of the evaluating neurosurgeon. No radiographic or symptomatic criteria were used to define shunt failure. When shunt failure occurred, the operative report for the revision was reviewed, and the portion of the shunt that was found to have failed at the time of surgery was documented. Proximal failure was considered failure of the ventricular catheter, while distal failure was considered failure of the valve or distal catheter.

\section{Statistical Analysis}

Statistical analysis was performed using commercially available software (SPSS version 21, IBM Corp.). All categorical data were assessed using the chi-square test. Multivariate logistic regression was used to assess independent association of our variables of interest with catheter placement accuracy. For purposes of multivariate logistic regression, the following were considered to be dichotomous variables: accuracy of placement (accurate/ inaccurate), institution (Michigan/Washington), side (left/ right), method of placement (guided placement including ultrasound and stereotactic neuronavigation/freehand). Age, Evans ratio, and bifrontal ventricular span were considered continuous variables. Statistical significance was considered to be $\mathrm{p}<0.05$.

\section{Results}

During the study period, 299 patients underwent placement of a parietooccipital ventricular catheter as part of a CSF shunt system; 42 patients were excluded due to lack of either pre- or postoperative imaging. Table 1 shows demographic details of the included cohort of 257 patients. The patients were nearly evenly split between the 2 participating institutions and nearly evenly split male versus female. The mean age of the included patients was 23 years. Intraoperative guidance (either ultrasound or stereotactic neuronavigation) was used in $39.3 \%$ of cases. The catheter was placed accurately in $72.8 \%$ of cases. Accurate catheter placement occurred in 4 of $8(50.0 \%)$ 
TABLE 1. Patient demographics*

\begin{tabular}{lc}
\hline \multicolumn{1}{c}{ Characteristic } & Value \\
\hline Total no. of patients & 257 \\
\hline Mean age, yrs & $23.0 \pm 26.9$ \\
\hline Patient population & $110(42.8)$ \\
\hline Adult & $147(57.2)$ \\
\hline Pediatric & \\
\hline Institution & $134(52.1)$ \\
\hline University of Michigan & $123(47.9)$ \\
\hline Washington University in St. Louis & $126(49.0)$ \\
\hline Sex & $131(51.0)$ \\
\hline Male & $0.36 \pm 0.11$ \\
\hline Female & $43.1 \pm 16.3$ \\
\hline Mean Evans ratio & $195(75.9)$ \\
\hline Mean bifrontal span, mm & $62(24.1)$ \\
\hline Operative side & $101(39.3)$ \\
\hline Right & $187(72.8)$ \\
\hline Left &
\end{tabular}

* Values are presented as the number of patients (\%) unless indicated otherwise. Mean values are presented as the mean \pm SD.

cases in which ultrasonography was used, 78 of $93(83.9 \%)$ cases in which stereotactic neuronavigation was used, and 105 of $156(67.3 \%)$ cases in which the freehand technique based on anatomical landmarks was used.

Bivariate comparisons were made between the group with accurate catheter placement and the group with inaccurate catheter placement (Table 2). The groups differed in mean age, preoperative Evans ratio, and whether intraoperative guidance was used. The mean age for those with accurate catheter placement was 20.4 years versus 30.0 years for those with inaccurate placement $(\mathrm{p}=0.010)$. The Evans ratio was slightly higher for those with inaccurate placement (0.38 inaccurate vs 0.35 accurate; $p=$ $0.049)$. When intraoperative guidance was used, there was a higher rate of accurate catheter placement $(81.2 \%)$ versus when the freehand technique was used $(67.3 \% ; \mathrm{p}=0.036)$.

Logistic regression was then used to create predictive models for the dichotomous outcome of accurate versus inaccurate catheter placement (Table 3). In univariate logistic regression models, both age and intraoperative guidance were associated with accuracy of catheter placement. Younger age was associated with an increased likelihood of accurate catheter placement, while the use of intraoperative guidance was also associated with accurate catheter placement. We planned a priori to include all variables with $\mathrm{p}<0.20$ in univariate analysis in a subsequent multivariate analysis. Based on this criterion, variables included in our multivariate analysis were age, institution, Evans ratio, side of catheter placement, and use of intraoperative guidance. After multivariate analysis, both age and use of intraoperative guidance remained significantly associated with accuracy of catheter placement. For every year of increase in age, patients were 0.983 times as likely to have an accurate catheter placement (95\% CI 0.971-0.995).
TABLE 2. Bivariate statistical comparison of accurate versus inaccurate ventricular catheter placement*

\begin{tabular}{|c|c|c|c|}
\hline Variable & $\begin{array}{l}\text { Accurate } \\
(n=187)\end{array}$ & $\begin{array}{l}\text { Inaccurate } \\
(\mathrm{n}=70)\end{array}$ & $\begin{array}{c}\mathrm{p} \\
\text { Value }\end{array}$ \\
\hline Mean age, yrs & $20.4 \pm 25.0$ & $30.0 \pm 30.5$ & 0.010 \\
\hline Patient population & & & 0.087 \\
\hline Adult & $74(67.3)$ & $36(32.7)$ & \\
\hline Pediatric & $113(76.9)$ & $34(23.1)$ & \\
\hline Institution & & & 0.123 \\
\hline University of Michigan & $92(68.7)$ & $42(31.3)$ & \\
\hline $\begin{array}{l}\text { Washington University } \\
\text { in St. Louis }\end{array}$ & $95(77.2)$ & $28(22.8)$ & \\
\hline Sex & & & 0.226 \\
\hline Male & $96(76.2)$ & $30(23.8)$ & \\
\hline Female & $91(69.5)$ & $40(30.5)$ & \\
\hline Mean Evans ratio & $0.35 \pm 0.11$ & $0.38 \pm 0.12$ & 0.049 \\
\hline Mean bifrontal span, mm & $42.5 \pm 17.0$ & $45.0 \pm 14.3$ & 0.276 \\
\hline Operative side & & & 0.054 \\
\hline Right & $136(69.7)$ & $59(30.3)$ & \\
\hline Left & $51(82.3)$ & $11(17.7)$ & \\
\hline Intraop guidance & & & 0.036 \\
\hline Yes & $82(81.2)$ & $19(18.8)$ & \\
\hline No & $105(67.3)$ & $51(32.7)$ & \\
\hline
\end{tabular}

* Values are presented as the number of patients (\%) unless indicated otherwise. Mean values are presented as the mean \pm SD.

When intraoperative guidance was used, accurate catheter placement was 2.809 times as likely as when intraoperative guidance was not used (95\% CI 1.406-5.618).

During the study period, 108 patients $(42.0 \%)$ experienced shunt failure, 79 patients $(30.7 \%)$ had failure involving the proximal catheter, and 53 patients (20.6\%) had distal failure (valve or distal catheter). Intraoperative guidance was used for 101 patients; 20 (19.8\%) of these patients experienced a proximal shunt failure. Among the 156 patients whose surgeries were performed without intraoperative guidance, $59(37.8 \%)$ had a proximal failure. Catheter placement was accurate in 187 patients; 52 $(27.8 \%)$ of these patients experienced a proximal shunt failure. Among the 70 patients with inaccurate catheter placement, 27 (38.6\%) had a proximal shunt failure. We next performed a logistic regression analysis to create predictive models of proximal failure (Table 4). In univariate analysis, age, preoperative Evans ratio, accurate catheter placement, and the use of intraoperative guidance were associated with the likelihood of proximal catheter failure. Again, we planned a priori to include all variables with $\mathrm{p}$ $<0.20$ in univariate analysis in a subsequent multivariate analysis. Based on this criterion, variables included in our multivariate analysis were age, preoperative Evans ratio, accurate catheter placement, and intraoperative guidance. After multivariate analysis, age, accurate catheter placement, and intraoperative guidance remained significantly associated with the likelihood of proximal catheter failure. For every year of increased age, patients were 0.978 times as likely to be proximal shunt failure free (95\% CI $0.966-0.990)$. When intraoperative guidance was used, 
TABLE 3. Univariate and multivariate logistic regression models of variables predicting accurate ventricular catheter placement

\begin{tabular}{lcccc}
\hline \multicolumn{1}{c}{ Variable } & Univariate OR $(95 \% \mathrm{Cl})$ & $\mathrm{p}$ Value & Multivariate OR $(95 \% \mathrm{Cl})$ & $\mathrm{p} \mathrm{Value}$ \\
\hline Age & $0.987(0.977-0.997)$ & 0.011 & $0.983(0.971-0.995)$ & 0.005 \\
\hline University of Michigan & $0.646(0.370-1.127)$ & 0.124 & $0.677(0.355-1.289)$ & 0.235 \\
\hline Female & $0.711(0.409-1.237)$ & 0.227 & & 0.111 \\
\hline Evans ratio & $0.089(0.008-1.015)$ & 0.051 & $0.103(0.006-1.681)$ & \\
\hline Bifrontal span & $0.991(0.975-1.007)$ & 0.281 & & 0.084 \\
\hline Left side placement & $2.011(0.979-4.131)$ & 0.057 & $1.934(0.915-4.085)$ & 0.016 \\
\hline Intraop guidance & $2.096(1.149-3.817)$ & 0.016 & $2.809(1.406-5.618)$ & \\
\hline
\end{tabular}

patients were 2.485 times as likely to be free from proximal catheter failure (95\% CI 1.227-5.032). Accurate catheter placement made patients 3.424 times as likely to be free from proximal catheter failure (95\% CI 1.796-6.524).

\section{Discussion}

CSF diversion is one of the most commonly performed neurosurgical operations. A variety of entry points are commonly employed for the ventricular catheter, including frontal and parietooccipital locations. Shunt failure is common. ${ }^{8}$ We have previously shown that the use of intraoperative guidance with either stereotactic neuronavigation or ultrasonography improves the accuracy of ventricular catheter placement and also reduces the rate of proximal catheter failure. ${ }^{12}$ However, the majority of patients included in that study had catheters placed via a frontal approach, limiting the generalizability to patients undergoing parietooccipital catheter placement. We hypothesized that a similar observation would be made for parietooccipital catheters, with intraoperative guidance improving the accuracy of catheter placement and reducing proximal failure. To address this hypothesis, we performed a multiinstitutional, retrospective, cohort study that included both adult and pediatric patients undergoing parietooccipital catheter placement as part of a CSF shunt system. We found that the use of intraoperative guidance significantly increased the likelihood of accurate catheter placement and also significantly decreased the likelihood of proximal catheter failure during the study period.

Frameless stereotaxy has previously been shown to be an effective adjunct for accurate ventricular catheter placement. ${ }^{1,12}$ However, this is the first study to directly compare intraoperative guidance to freehand placement of parietooccipital catheters. The overall rate of accurate catheter placement in our study was $72.8 \%$. When intraoperative guidance was used, $81.2 \%$ of catheters were placed accurately compared with only $67.3 \%$ of catheters placed by freehand technique. While this is a significant increase in accuracy, the increase is not as significant as what was observed in our previous study of predominantly frontal catheters. In that study, $88.6 \%$ of catheters placed with intraoperative guidance were placed accurately. ${ }^{12}$ This may suggest increased inaccuracy of intraoperative guidance for catheters placed with a posterior approach. Anecdotally, we have found that stereotactic neuronavigation systems seem to be less accurate when used for other purposes (e.g., tumors) in the posterior part of the head. In the current study, the majority of intraoperative guidance came in the form of frameless stereotaxy (93 of 101 guided catheters), while the minority were placed using ultrasonic guidance ( 8 of 101 guided catheters). Thus, this study is most directly generalizable to intraoperative guidance using frameless stereotaxy.

We have observed that a commonly employed technique during CSF shunt operations is to use intraoperative guidance for perceived "small" ventricles and to otherwise use the freehand technique for ventricular catheter placement. Our data argue against this approach, as ventricle size was not predictive of accurate catheter placement. In fact, in bivariate analysis, the Evans ratio for patients with inaccurate placement was actually higher than that for those with accurate placement. However, this was not borne out in logistic regression analysis, where neither Evans ratio nor biventricular span was predictive of accuracy. These data suggest that intraoperative guidance is useful

TABLE 4. Univariate and multivariate logistic regression models of variables predicting no proximal failure

\begin{tabular}{lcccc}
\hline \multicolumn{1}{c}{ Variable } & Univariate OR $(95 \% \mathrm{Cl})$ & $\mathrm{p}$ Value & Multivariate OR $(95 \% \mathrm{Cl})$ & $\mathrm{p} \mathrm{Value}$ \\
\hline Age & $0.978(0.966-0.990)$ & $<0.001$ & $0.983(0.970-0.995)$ & 0.008 \\
\hline University of Michigan & $0.917(0.539-1.557)$ & 0.747 & & \\
\hline Female & $1.135(0.668-1.930)$ & 0.640 & & 0.407 \\
\hline Evans ratio & $20.696(1.903-225.100)$ & 0.013 & $2.937(0.230-37.527)$ & \\
\hline Bifrontal span & $1.004(0.988-1.020)$ & 0.663 & & 0.011 \\
\hline Left side placement & $0.729(0.383-1.387)$ & 0.335 & & 0.009 \\
\hline Intraop guidance & $3.877(2.027-7.415)$ & $<0.001$ & $2.485(1.227-5.032)$ & $3.424(1.796-6.524)$ \\
\hline Accurate placement & $3.260(1.830-5.810)$ & 0.013 & & \\
\hline
\end{tabular}


for improving accuracy of ventricular catheter placement regardless of ventricle size. The only other factor found to be predictive of accurate catheter placement was age, with increasing age being associated with an increased likelihood of inaccuracy. An explanation for this observation is unclear.

An overall rate of inaccuracy of approximately $27 \%$ and a rate of approximately $33 \%$ when the freehand technique is employed seems extraordinarily and unacceptably high. However, these data combined with previous data suggest that we underestimate the inaccuracy of ventricular catheter placement. In our previous study, we found a rate of $45 \%$ inaccurate placement using the freehand technique. ${ }^{12}$ Lind et al. previously found that $44 \%$ of catheters were placed inaccurately versus $47 \%$ in a study by Theodosopoulos et al. (with accuracy defined by the same standards as our current study). ${ }^{5,10}$ This underestimation of inaccurate catheter placement may lead to underestimation of the need for intraoperative guidance. By further adding to the data suggesting a high rate of inaccurate catheter placement, we hope to increase recognition of the value of intraoperative guidance.

The most important follow-up question is whether accuracy, or more directly the use of intraoperative guidance, has an effect on shunt failure. Since the accuracy of ventricular catheter placement is unlikely to have an effect on the shunt valve or function of the distal catheter, we chose to specifically analyze the effect of intraoperative guidance on proximal catheter failure, the portion of the shunt system most likely to be directly affected by intraoperative guidance. During the study period, $42.0 \%$ of patients experienced a shunt failure, $30.7 \%$ of patients experienced a failure involving the proximal catheter, and $20.6 \%$ of patients experienced a failure involving the valve or distal catheter. This is in line with previous reports suggesting that approximately one-third of patients will experience failure of the CSF shunt. ${ }^{8}$ Among the variables analyzed in the current study, age, accurate catheter placement, and intraoperative guidance were associated with proximal catheter failure. Older patients were less likely to be free of proximal failure, while the use of intraoperative guidance and accurate catheter placement significantly reduced the rate of proximal catheter failure. Thus, these data suggest that the improvement in accuracy of ventricular catheter placement achieved using intraoperative guidance translates into a reduction in proximal catheter failure.

One potential limitation to the study is our definition of failure. We used a surgical definition of shunt failure with shunt revision/exploration surgery being the sole definition of shunt failure. This overlooks partial failures or asymptomatic failures. While our definition may underestimate shunt failure, we still believe that the definition used is clinically relevant. It may be more appropriate to say that our study suggests that improvement in accuracy of ventricular catheter placement achieved using intraoperative guidance translates into a reduction in revisions of CSF shunts due to proximal catheter failure.

Given that the overall rate of shunt failure is so high, we believe that every effort should be made to optimize the procedure in such a way as to minimize the risk of failure. This means that each component of the shunt sys- tem, from the technology to the technical details of surgery, should be studied in an effort to improve the overall system. Combining our current data with our previous data as well as with data from Villavicencio et al., optimal ventricular catheter placement seems to reduce the likelihood of proximal shunt failure. ${ }^{11,12}$ There is a growing body of literature indicating that laparoscopic assistance for placement of a peritoneal catheter (the most common terminus in CSF shunting operations) may reduce the rate of distal catheter failure. ${ }^{6,79}$ Furthermore, there are some data to suggest that the use of programmable valves may reduce the rate of failure/revision in comparison with fixed pressure valves. ${ }^{2}$ While we do not specifically advocate the use of laparoscopic placement or programmable valves, we do believe it is important to continue to study ways of improving CSF diversion to lessen the risk of shunt failure.

The optimal definition for accurate ventricular catheter placement is not defined. We used a simple dichotomous definition in this study based on the approach of the catheter and the location of the tip of the catheter. This is similar to the definition used in our previous study. ${ }^{12}$ However, others have previously used differing definitions. For example, Hayhurst and colleagues defined accuracy based on the tip of the catheter relative to the ventricular walls and choroid: free-floating versus touching the ventricular wall or choroid versus intraparenchymal. ${ }^{3}$ While their definition intuitively makes sense if the working hypothesis for proximal shunt failure is that it occurs most frequently due to obstruction by the choroid plexus, it ignores the structure of the ventricular catheter with drainage occurring along the entire distal $3 \mathrm{~cm}$ of the catheter. Thus, it is not clear that a more complex definition of accuracy such as that used by Hayhurst offers advantages over our more simplified definition, but the optimal definition is not clearly defined.

\section{Conclusions}

To our knowledge, this is the first study to compare accuracy of parietooccipital ventricular catheter placement and proximal shunt failure rates between catheters placed using intraoperative guidance versus those placed using the freehand technique and standard anatomical landmarks. The use of intraoperative guidance significantly improved the rate of accurate catheter placement and reduced the rate of proximal shunt failure. Ventricle size was not associated with the accuracy of catheter placement. In a procedure that is fraught with a high failure rate, we believe these data support the routine use of intraoperative guidance to improve parietooccipital catheter accuracy and to reduce proximal shunt failure.

\section{References}

1. Azeem SS, Origitano TC: Ventricular catheter placement with a frameless neuronavigational system: a 1-year experience. Neurosurgery 60 (4 Suppl 2):243-248, 2007

2. Farahmand D, Hilmarsson H, Högfeldt M, Tisell M: Perioperative risk factors for short term shunt revisions in adult hydrocephalus patients. J Neurol Neurosurg Psychiatry 80:1248-1253, 2009

3. Hayhurst C, Beems T, Jenkinson MD, Byrne P, Clark S, Kan- 
dasamy J, et al: Effect of electromagnetic-navigated shunt placement on failure rates: a prospective multicenter study. $\mathbf{J}$ Neurosurg 113:1273-1278, 2010

4. Korinek AM, Fulla-Oller L, Boch AL, Golmard JL, Hadiji B, Puybasset L: Morbidity of ventricular cerebrospinal fluid shunt surgery in adults: an 8-year study. Neurosurgery 68:985-995, 2011

5. Lind CR, Tsai AM, Lind CJ, Law AJ: Ventricular catheter placement accuracy in non-stereotactic shunt surgery for hydrocephalus. J Clin Neurosci 16:918-920, 2009

6. Naftel RP, Argo JL, Shannon CN, Taylor TH, Tubbs RS, Clements RH, et al: Laparoscopic versus open insertion of the peritoneal catheter in ventriculoperitoneal shunt placement: review of 810 consecutive cases. J Neurosurg 115:151-158, 2011

7. Phan S, Liao J, Jia F, Maharaj M, Reddy R, Mobbs RJ, et al: Laparotomy vs minimally invasive laparoscopic ventriculoperitoneal shunt placement for hydrocephalus: A systematic review and meta-analysis. Clin Neurol Neurosurg 140:2632, 2016

8. Reddy GK, Bollam P, Shi R, Guthikonda B, Nanda A: Management of adult hydrocephalus with ventriculoperitoneal shunts: long-term single-institution experience. Neurosurgery 69:774-781, 2011

9. Schucht P, Banz V, Trochsler M, Iff S, Krähenbühl AK, Reinert M, et al: Laparoscopically assisted ventriculoperitoneal shunt placement: a prospective randomized controlled trial. J Neurosurg 122:1058-1067, 2015

10. Theodosopoulos PV, Abosch A, McDermott MW: Intraoperative fiber-optic endoscopy for ventricular catheter insertion. Can J Neurol Sci 28:56-60, 2001
11. Villavicencio AT, Leveque JC, McGirt MJ, Hopkins JS, Fuchs HE, George TM: Comparison of revision rates following endoscopically versus nonendoscopically placed ventricular shunt catheters. Surg Neurol 59:375-380, 2003

12. Wilson TJ, Stetler WR Jr, Al-Holou WN, Sullivan SE: Comparison of the accuracy of ventricular catheter placement using freehand placement, ultrasonic guidance, and stereotactic neuronavigation. J Neurosurg 119:66-70, 2013

\section{Disclosures}

The authors report no conflict of interest concerning the materials or methods used in this study or the findings specified in this paper.

\section{Author Contributions}

Conception and design: Sullivan, Smyth. Acquisition of data: all authors. Analysis and interpretation of data: all authors. Drafting the article: Wilson. Critically revising the article: Wilson, Smyth. Reviewed submitted version of manuscript: all authors. Approved the final version of the manuscript on behalf of all authors: Sullivan. Study supervision: Sullivan, Smyth.

\section{Correspondence}

Stephen E. Sullivan, Department of Neurosurgery, University of Michigan, 1500 E Medical Center Dr., Rm. 3552 TC, Ann Arbor, MI 48109-5338. email: ssulliva@med.umich.edu. 